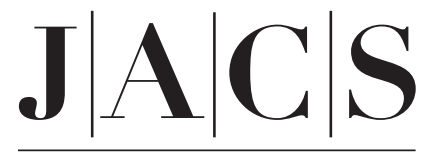

A R T I C L E S

Published on Web 12/24/2005

\title{
Multiple Wurtzite Twinning in CdTe Nanocrystals Induced by Methylphosphonic Acid
}

\author{
Luigi Carbone, ${ }^{\dagger}$ Stefan Kudera, ${ }^{\ddagger}$ Elvio Carlino, ${ }^{\S}$ Wolfgang J. Parak, ${ }^{\ddagger}$ \\ Cinzia Giannini, ${ }^{\perp}$ Roberto Cingolani, ${ }^{\dagger}$ and Liberato Manna*, $\dagger$ \\ Contribution from the National Nanotechnology Laboratory of CNR-INFM, 73100 Lecce, Italy, \\ Center for Nanoscience, Ludwig Maximilians Universität, München, Germany, \\ TASC-INFM-CNR National Laboratory, Area Science Park - Basovizza, 34012 Trieste, Italy, \\ and CNR-Istituto di Cristallografia (IC), via Amendola 122/O, 70126 Bari, Italy
}

Received July 21, 2005; E-mail: liberato.manna@unile.it

\begin{abstract}
Branching in semiconductor nanocrystals, which leads to tetrapods and to more complex architectures, is the subject of intensive investigation. Here we support the model according to which branching in CdTe nanocrystals is driven by the formation of multiple wurtzite twins. This is in contrast to previous models for this material. We found that twinning, as well as anisotropic growth, can be triggered by the presence of suitable molecules, such as for instance methylphosphonic acid. In the case of CdTe nanocrystals, we designed a robust growth scheme in which the variation of a single parameter (the concentration of methylphosphonic acid in solution) leads to the controlled formation of nanocrystals with shapes ranging from spheres to anisotropic structures with varying level of branching, as both twinning and anisotropic growth are progressively favored. We believe that these concepts can be extended to other nanocrystal systems.
\end{abstract}

\section{Introduction}

The synthesis of nanocrystals with complex shapes is an exciting development in nanoscience ${ }^{1}$ as it paves the way to materials with tailored, geometry-dependent properties and to new concepts of self-assembly arising from the mutual interaction of such nanocrystals. The tetrapod, for instance (Figure $1)$, is a shape that occurs frequently in several materials $\left(\mathrm{ZnO},{ }^{2-11} \mathrm{ZnSe},{ }^{12} \mathrm{ZnS},{ }^{13} \mathrm{CdS},{ }^{14-16} \mathrm{CdSe},{ }^{17} \mathrm{CdTe},{ }^{18-20} \mathrm{CuCl},{ }^{21}\right.$

† National Nanotechnology Laboratory of CNR-INFM.

$¥$ Center for Nanoscience, Ludwig Maximilians Universität.

$\S$ TASC-INFM-CNR National Laboratory.

${ }^{\perp}$ CNR-Istituto di Cristallografia (IC).

(1) Burda, C.; Chen, X. B.; Narayanan, R.; El-Sayed, M. A. Chem. Rev. 2005, $105,(4), 1025-1102$.

(2) Kitano, M.; Hamabe, T.; Maeda, S.; Okabe, T. J. Cryst. Growth 1991, $108,(1-2), 277-284$

(3) Fujii, M.; Iwanaga, H.; Ichihara, M.; Takeuchi, S. J. Cryst. Growth 1993, $128,(1-4), 1095-1098$.

(4) Takeuchi, S.; Iwanaga, H.; Fujii, M. Philos. Mag. A 1994, 69, (6), 11251129

(5) Nishio, K.; Isshiki, T.; Kitano, M.; Shiojiri, M. Philos. Mag. A 1997, 76, (4), 889-904

(6) Iwanaga, H.; Fujii, M.; Takeuchi, S. J. Cryst. Growth 1998, 183, (1-2), $190-195$.

(7) Dai, Y.; Zhang, Y.; Wang, Z. L. Solid State Commun. 2003, 126, (11), 629-633.

(8) Yan, H. Q.; He, R. R.; Pham, J.; Yang, P. D. Adv. Mater. 2003, 15, (5), $402-405$.

(9) Chen, Z.; Shan, Z. W.; Cao, M. S.; Lu, L.; Mao, S. X. Nanotechnology 2004, 15, (3), 365-369.

(10) Yu, W. D.; Li, X. M.; Gao, X. D. Cryst. Growth Des. 2005, 5, (1), 151155 .

(11) Wang, F. Z.; Ye, Z. Z.; Ma, D. W.; Zhu, L. P.; Zhuge, F. J. Cryst. Growth 2005, 274, (3-4), 447-452

(12) Hu, J. Q.; Bando, Y. S.; Golberg, D. Small 2005, 1, (1), 95-99.

(13) Zhu, Y. C.; Bando, Y.; Xue, D. F.; Golberg, D. J. Am. Chem. Soc. 2003 $125,(52), 16196-16197$.

(14) Jun, Y. W.; Lee, S. M.; Kang, N. J.; Cheon, J. J. Am. Chem. Soc. 2001, $123,(21), 5150-5151$. and $\mathrm{Pt}^{22}$ ) and is formed by four arms that branch out at tetrahedral angles from a central region. Tetrapod-shaped nanocrystals present promising potential for building blocks in solar cells, ${ }^{23,24}$ as active components in nanoscale transistors, ${ }^{25}$ as field emitters, and might open up new possibilities to create unique architectures that cannot be realized with spherical or rod-shaped nanocrystals.

Understanding the mechanism of branching in colloidal nanocrystals is of fundamental importance, as this is a prerequisite for unfolding the detailed structure and the intrinsic electronic properties of these nanocrystals ${ }^{26}$ and for achieving a better control over the fabrication of more elaborate nanoparticle shapes. In the case of solution-grown nanocrystals of II-VI semiconductors, several reports have proposed the model of the tetrapod (Figure 1f) as formed by a sphalerite nucleus (Figure 1a), onto which wurtzite arms have developed by

(15) Chen, M.; Xie, Y.; Lu, J.; Xiong, Y. J.; Zhang, S. Y.; Qian, Y. T.; Liu, X. M. J. Mater. Chem. 2002, 12, (3), 748-753.

(16) Shen, G. Z.; Lee, C. J. Cryst. Growth Des. 2005, 5, (3), 1085-1089.

(17) Manna, L.; Scher, E. C.; Alivisatos, A. P. J. Am. Chem. Soc. 2000, 122, (51), $12700-12706$.

(18) Yu, W. W.; Wang, Y. A.; Peng, X. G. Chem. Mater. 2003, 15, (22), 43004308.

(19) Bunge, S. D.; Krueger, K. M.; Boyle, T. J.; Rodriguez, M. A.; Headley, T. J.; Colvin, V. L. J. Mater. Chem. 2003, 13, (7), 1705-1709.

(20) Manna, L.; Milliron, D. J.; Meisel, A.; Scher, E. C.; Alivisatos, A. P. Nat. Mater. 2003, 2, (6), 382-385.

(21) Li, Q.; Shao, M. W.; Yu, G. H.; Wu, J.; Li, F. Q.; Qian, Y. T. J. Mater. Chem. 2003, 13, (2), 424-427.

(22) Teng, X.; Yang, H. Nano Lett. 2005, 5, (5), 885-891.

(23) Huynh, W. U.; Dittmer, J. J.; Alivisatos, A. P. Science 2002, 295, (5564), $2425-2427$

(24) Sun, B. Q.; Marx, E.; Greenham, N. C. Nano Lett. 2003, 3, (7), 961-963.

(25) Cui, Y.; Banin, U.; Bjork, M. T.; Alivisatos, A. P. Nano Lett. 2005, 5, (7), $1519-1523$.

(26) Li, J. B.; Wang, L. W. Nano Lett. 2003, 3, (10), 1357-1363.

10.1021/ja054893c CCC: $\$ 33.50 \odot 2006$ American Chemical Society

748 a J. AM. CHEM. SOC. 2006, 128, 748-755 


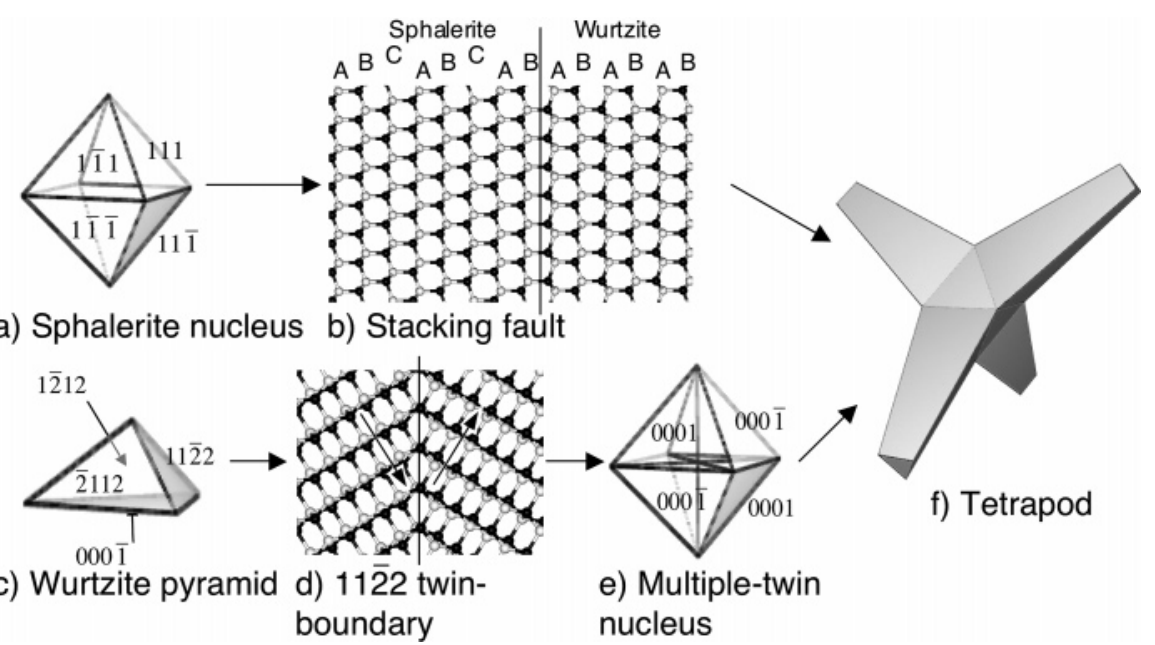

Figure 1. Two different models that rationalize the tetrapod shape. (a) A sphalerite nucleus with four equivalent $\{111\}$ facets and four equivalent $\{111\}$ facets. (b) By generation of stacking faults on the four fast growing facets, the growth on these facets would continue in the hexagonal phase, leading to a tetrapod shape (f). (e) A multiple-twin nucleus formed by eight pyramid-shaped wurtzite crystals (c). (d) Structural details of the (1122) twin boundary present in this type of nucleus. The lattice boundary is observed from the (1010) zone axis. The arrows in (d) indicate the direction of the polarity in the two wurtzite domains.

continuation of growth from four equivalent $\{111\}$ facets. This occurs through generation of stacking faults (Figure 1b). ${ }^{14,15,17,18,20}$ This model is plausible, since the coexistence of wurtzite and sphalerite phases in II-VI semiconductors is frequent, due to the low energy of formation of stacking faults in these materials. ${ }^{27,28}$ However, this model has not been fully proven thus far, due to the lack of detailed TEM investigations. Data from existing studies either do not allow for a unambiguous conclusion about the structure of the tetrapod because the central seed is too small to be clearly identified as a sphalerite domain, ${ }^{15}$ or can be interpreted by different models.

Another popular model that rationalizes the tetrapod shape (for instance, in $\mathrm{ZnO}$ and $\mathrm{ZnSe}{ }^{4-7,12,29,30}$ ) proposes that the initial nucleus is formed by eight wurtzite domains connected to each other through $(11 \overline{2} 2)$ twin boundaries (Figure 1c,e). For each couple of domains sharing a twin plane there is a head-to-tail configuration of the polarity (Figure 1d). This particular type of boundary has a low energy of formation (higher, however, than that of a stacking fault) because it does not involve the creation of dangling bonds and requires little lattice distortion. Ideally, the multiple-twin nucleus is then terminated by four (0001) and by four $(000 \overline{1})$ wurtzite facets. The growth rate between these two groups of facets is remarkably different; ${ }^{17,31}$ hence, four out of the eight domains making up the nucleus are "fast growing", and the remaining four are "slow growing". Therefore, the initial nucleus evolves to a tetrapod (Figure 1f). This more elaborate model has been supported by the statistical analysis of the inter-leg angles in $\mathrm{ZnO}$ tetrapods $^{6}$ (which agree with the angles that are generated by complete relaxation of the octahedral nucleus ${ }^{32}$ ) and has been confirmed in part also by transmission electron microscopy. ${ }^{7}$

(27) Takeuchi, S.; Suzuki, K.; Maeda, K., Philos. Mag. A 1984, 50, (2), 171178

(28) Yeh, C. Y.; Lu, Z. W.; Froyen, S.; Zunger, A. Phys. Rev. B 1992, 46, (16), 10086-10097.

(29) Iwanaga, H.; Fujii, M.; Takeuchi, S. J. Cryst. Growth 1993, 134, (3-4), $275-280$.

(30) Iwanaga, H.; Fujii, M.; Ichihara, M.; Takeuchi, S. J. Cryst. Growth 1994 $141,(1-2), 234-238$

(31) Kudera, S.; Carbone, L.; Casula, M. F.; Cingolani, R.; Falqui, A.; Snoeck, E.; Parak, W. J.; Manna, L. Nano Lett. 2005, 5, (3), 445-449.
The synthesis of tetrapod-shaped CdTe nanocrystals is well documented, ${ }^{18-20}$ and as such this material can be considered as a model system for studying the branching in nanocrystals. In the present work, we support the model according to which the branching in colloidal CdTe nanocrystals is due to the formation of multiple wurtzite twins, which we are able to trigger. The nanocrystals were synthesized at high temperatures in a mixture of surfactants. We show that a small molecule, such as methylphosphonic acid (MPA) is a powerful additive to control both branching and anisotropic growth. When no MPA was added, roughly spherical nanocrystals were always formed. When MPA was present, the shape of the synthesized nanocrystals varied from short, pear-like shapes to structures that were progressively more branched and that grew more anisotropically, depending on the concentration of MPA. Highresolution transmission electron microscopy (HRTEM) revealed that the branching region in tetrapods is characterized by the presence of multiple wurtzite domains. In structures with a number of arms lower than four this region could be studied in more detail and showed the occurrence of twins. In particular, the $11 \overline{2} 2$ twin boundary with a head-to-tail polarity configuration was identified clearly and unambiguously in nanocrystals in which a single arm departed from a multiple-twinned region. Other types of wurtzite twins could be present in CdTe nanocrystals, although among them the $11 \overline{2} 2$ twin boundary has the lowest energy of formation and therefore the highest probability of occurrence. Twinned wurtzite regions were also observed by HRTEM in tetrapods. Due to the more complicated geometry of the latter experiments, the HRTEM images were compared to the relevant simulations of the twinned core of a branched nanocrystal generated by using atomistic models based on multiple wurtzite domains joined by $11 \overline{2} 2$ twin boundaries. Therefore, we are confident that these data can explain the tetrapod shape in the light of the multiple-twin model, although

(32) The formation of the octa-twin requires in fact the accumulation of a large strain if the various pyramids have to join to form a perfect octahedron. However, past a certain critical size for the nucleus, this strain must be released by the generation of cracks along the twin boundaries, and this disrupts the perfect tetrapod geometry, which has indeed never been observed in actual samples. 
we cannot rule out that some branched nanocrystals form as a consequence of wurtzite-sphalerite polymorphism. We believe that these concepts can be applied also to other nanocrystalline materials that show similar shapes in solution (i.e., CdSe and $\mathrm{CdS}$ ), and as such they can deepen our knowledge on the formation of these systems.

\section{Experimental Section}

Chemicals and Stock Solutions. Cadmium oxide (CdO, 99.999\%), trioctylphosphine oxide (TOPO 99\%), trioctylphosphine (TOP, 97\%), and tellurium (99.999\%) were purchased from Strem Chemicals. Octadecylphosphonic acid (ODPA, 99\%) was purchased from Polycarbon Industries. Methylphosphonic acid (MPA, 98\%) was purchased from Aldrich. All solvents used were anhydrous and were manipulated in a nitrogen drybox. A stock solution of MPA in distilled water (18.2 $\mathrm{M} \Omega$ ) was prepared as $2.9 \%$ in weight. A solution of Te in TOP $(10 \%$ in weight) was prepared by mixing $1 \mathrm{~g}$ of Te powder with $9 \mathrm{~g}$ of TOP under nitrogen, and then by heating the mixture to $250{ }^{\circ} \mathrm{C}$ for several hours until the tellurium was completely dissolved. When cooled to room temperature, the resulting yellow solution was centrifuged at 4000 rpm for several hours to separate it from undissolved products, which were collected as a tiny, white-grayish precipitate.

Synthesis of CdTe Nanocrystals. All syntheses were carried out under nitrogen atmosphere. In all experiments, the molar ratio of cadmium to phosphonic acids (ODPA + MPA) was set to 1.0:3.2, the molar ratio of cadmium to tellurium was set to 3.5:1.0, and the total amount of surfactants (TOPO + ODPA + MPA) was kept to 4.1-4.2 g. The only parameter that was changed was the MPA:ODPA molar ratio, which was varied from 0 (only ODPA present) to 0.18 . As an example of a typical synthesis of CdTe nanocrystals that yielded mainly tetrapods, $52 \mathrm{mg}$ of $\mathrm{CdO}$ were mixed in a $50 \mathrm{~mL}$ three-neck flask with $3.75 \mathrm{~g}$ of TOPO, $0.385 \mathrm{~g}$ of ODPA, and $0.358 \mathrm{~g}$ of the MPA solution. The resulting solution was pumped to vacuum for $30 \mathrm{~min}$ at $100{ }^{\circ} \mathrm{C}$. To decompose the $\mathrm{CdO}$, the solution was heated to $350{ }^{\circ} \mathrm{C}$ under nitrogen until it turned completely transparent and clear. The temperature was stabilized at $320{ }^{\circ} \mathrm{C}$ and $0.146 \mathrm{~g}$ of the Te:TOP solution, diluted with additional TOP (to a total weight of $0.450 \mathrm{~g}$ ) was quickly injected in the flask. In all syntheses, the crystals were allowed to grow for $10 \mathrm{~min}$ at $320^{\circ} \mathrm{C}$, after which the reaction was stopped by removing the heating mantle. When the solution was cooled to $50{ }^{\circ} \mathrm{C}, 3-5 \mathrm{~mL}$ of chloroform was added to it and the whole content of the flask was transferred in the drybox. The nanocrystals were purified by repeated precipitation with methanol and solubilization in chloroform.

Transmission Electron microscopy (TEM). The samples were prepared by dispersing dilute solutions of nanocrystals onto carboncoated copper grids. Low-magnification TEM images were recorded on a JEOL Jem 1011 microscope operated at $100 \mathrm{KV}$. Further TEM and scanning TEM (STEM) experiments were performed at room temperature on a JEOL JEM 2010F UHR TEM/STEM electron microscope, with field-emission gun, operating at $200 \mathrm{kV}(\lambda=0.0025$ $\mathrm{nm})$, with a measured spherical aberration coefficient $C_{\mathrm{s}}$ of $0.47 \pm$ $0.01 \mathrm{~mm}$ and producing a relevant interpretable resolution limit in HRTEM of $0.19 \mathrm{~nm} .{ }^{33}$ For Z-contrast imaging, the STEM attachment was equipped with a YAP high angle annular dark field (HAADF) detector. The theoretical resolution achievable in Z-contrast mode with the available electron optics is $0.126 \mathrm{~nm} .{ }^{34} \mathrm{Z}$-contrast micrographs were recorded with a collection angle $84 \leq 2 \theta \leq 224 \mathrm{mrad}$. TEM/STEM observations were performed to identify unambiguously the crystal Bravais lattice of the branching region and thus to assess whether this had wurtzite or sphalerite structure. For this purpose, several nanocrystals were imaged which were oriented along some particular zone

(33) Spence, J. C. H. Experimental High-Resolution Electron Microscopy, 2nd ed.; Oxford University Press: Oxford, 1988; p 87.

(34) Pennycook, S. J. Advances in Imaging and Electron Physics; Academic: New York, 2002; Vol. 123, p 140. axes that allowed distinguishing between the two types of lattices from the analysis of their diffraction patterns. This differentiation is straightforward: for instance if the orientation of the particles is such that the symmetry of their diffraction pattern is characterized by six diffraction spots placed on the vertex of a regular hexagon. This pattern symmetry is peculiar of both the $\langle 111\rangle$ zone axis of the sphalerite structure and of the $\langle 0001\rangle$ zone axis of the wurtzite structure. However, in the sphalerite case the diffraction spots are due to the six $\{\overline{2} 02\}$ families of planes, which are spaced by $0.229 \mathrm{~nm}$, whereas in the wurtzite case they are due to both the $\{100\}$ and the $\{\overline{1} 10\}$ families of planes, which are spaced by $0.398 \mathrm{~nm}$. Hence, by measuring the lattice spacings, the two structures can be distinguished from each other.

X-ray Diffraction (XRD). XRD measurements were performed with a D8 Discover-Bruker diffractometer equipped with a $3 \mathrm{~kW}$ ceramic tube with a copper anode, a Goebel type parabolic mirror and twobounces monochromator (V Groove) as primary optics, and a $\mathrm{NaI}(\mathrm{Tl})$ scintillator detector. A coupled $\theta-2 \theta$ movement was chosen for data collection. Concentrated nanocrystal solutions were spread on top of a silicon substrate and then the sample was allowed to dry and was measured in reflection geometry.

Calculations. The simulations of the HRTEM images were carried out in the framework of the Multislice approach. ${ }^{35}$ This approach allows managing supercells containing thousands of atoms, which are necessary to correctly describe the central region of the tetrapods. In practice, the simulations were performed using the program MACTEMPAS (www.totalresolution.com).

$\mathrm{Ab}$ initio, DFT calculations of the twin formation energy were performed using the PEtot software, which is based on a plane wave expansion and uses the density functional theory. ${ }^{36}$ The exchangecorrelation functional was evaluated within the local density approximation (LDA). ${ }^{37,38}$ Pseudopotentials ${ }^{39}$ for $\mathrm{Cd}$ and Te atoms were generated according to the improved Troullier and Martins method, ${ }^{40}$ with the Ceperly-Alder exchange-correlation function ${ }^{41}$ and using 12 valence electrons for $\mathrm{Cd}\left(4 \mathrm{~d}^{10} 5 \mathrm{~s}^{2}\right)$ and 6 valence electrons for Te $\left(5 \mathrm{~s}^{2} 5 \mathrm{p}^{4}\right)$ without core correction, respectively. For $\mathrm{Cd}$, the $5 \mathrm{~s}$ electrons were used for the local part of the pseudopotential. The plane wave cutoff energy for the wave function was set to $45 \mathrm{Ryd}$, while the cutoff energy for the charge density was set to 150 Ryd. The cell used to model the twin boundary contained 64 atoms (for a total of 576 valence electrons) and was adapted from the cell used by Yan and co-workers to model the same type of defect in $\mathrm{ZnO}{ }^{42}$ Eight symmetry-reduced special k-points for the supercell calculations were used. The atomic positions were optimized to minimize the forces acting on the atoms.

\section{Results}

The results of the syntheses performed at MPA:ODPA molar ratios ranging from 0 to 0.18 are summarized in Figure 2. The clear trend here is that the higher the MPA:ODPA ratio, the more branched and elongated the nanocrystals grow. A statistical analysis was also carried out on the TEM images of these samples to determine the average number of branches per nanocrystal (Figure 2, inset). From this analysis and from an overall glance at Figure 2, one can deduct that tetrapods (four branches per nanocrystal) are best synthesized at MPA:ODPA ratios around $0.08-0.09$ (Figure 2d,e). Slightly lower ratios

(35) Goodman, P.; Moodie, A. F. Acta Crystallogr., Sect. A 1974, A30, 280290.

(36) Wang, L. W. Petot: A Parallel Plane Wave Pseudopotential Program for Atomistic Total Energy Calculation Based on Density Functional Theory, Version 2; Berkeley, 2004.

(37) Hohenberg, P.; Kohn, W. Phys. Rev. 1964, 136, 864B.

(38) Kohn, W.; Sham, L. J. Phys. Rev. 1965, 140, 1133A.

(39) Heine, V. In Solid State Physics; Ehrenreich, H., Seitz, F., Turnbull, D. Eds.; Academic Press: New York, 1970; Vol. 24, pp 1-36.

(40) Troullier, N.; Martins, J. L. Phys. Rev. B 1991, 43, (3), 1993-2006.

(41) Perdew, J. P.; Zunger, A. Phys. Rev. B 1981, 23, (10), 5048-5079.

(42) Yan, Y. F.; Al-Jassim, M. M.; Chisholm, M. F.; Boatner, L. A.; Pennycook, S. J.; Oxley, M. Phys. Rev. B 2005, 71, (4), art. no.-041309. 

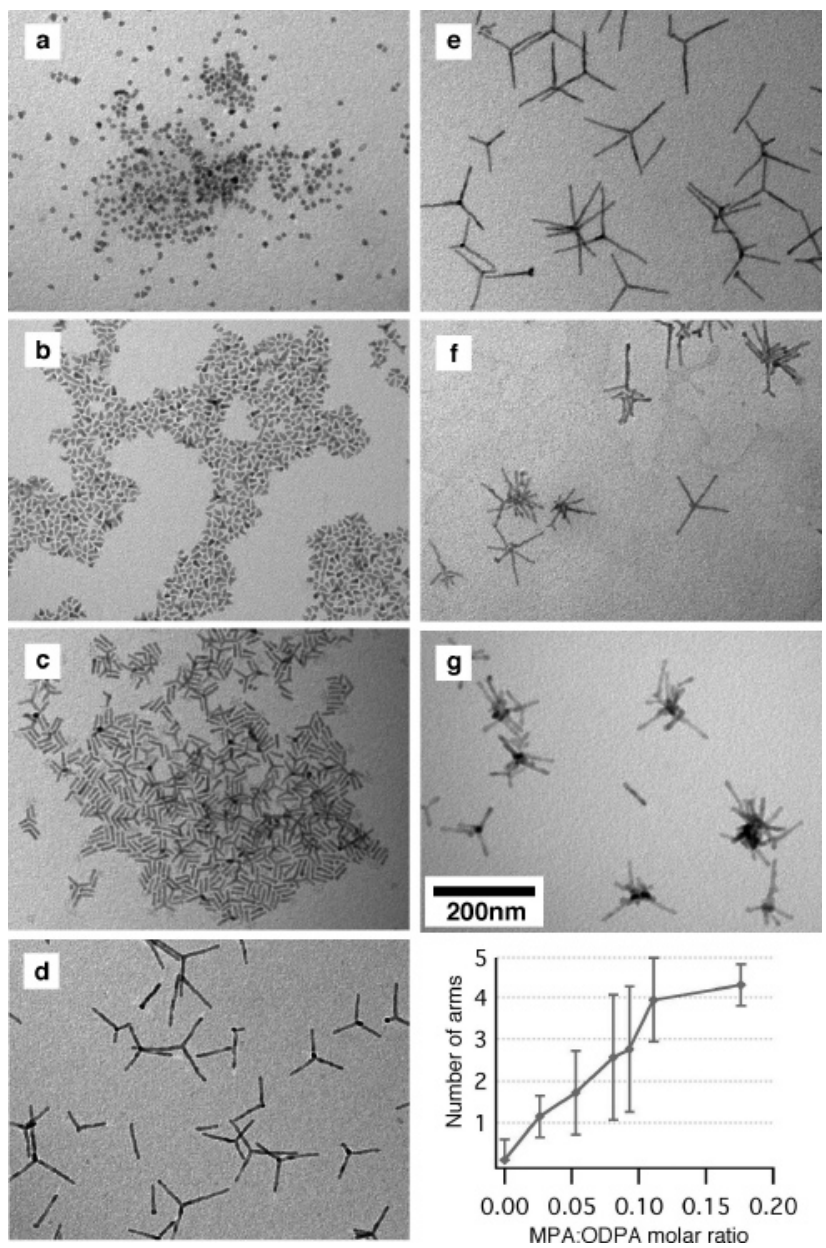

Figure 2. Low-magnification TEM images and results of statistical analysis of a series of samples prepared at increasing MPA:ODPA molar ratios. (a) 0, (b) 0.026 , (c) 0.053, (d) 0.081, (e) 0.093, (f) 0.111, and (g) 0.176. All syntheses were run for $10 \mathrm{~min}$. In the statistical analysis, the number of branches for each nanocrystal was assigned as follows: zero for spherical nanocrystals; $1,2,3$, and 4, for rods, dipods, tripods, and tetrapods, respectively, while multibranched objects were simply assigned five arms. The large errors are due to the fact that often the determination of the number of branches in a nanocrystal is not straightforward, especially for highly branched structures. Also, highly branched structures are not weighted adequately by the analysis, leading to an artificial shift toward smaller numbers on average.

(0.05-0.08) will tend to yield more regular tetrapods but accompanied with a large number of nanocrystals having a lower number of arms (rods, dipods, tripods).

When only ODPA was used in the syntheses, almost spherical nanocrystals were obtained (Figure 2a and Figure 3a,b). X-ray diffraction (Supporting Information) indicated that this sample contained a mixture of wurtzite and sphalerite nanocrystals. The formation of branched nanocrystals was rarely observed. On the other hand, the nanocrystals showed a remarkable occurrence of stacking faults. In Figure 3c,d two representative nanocrystals from this sample are shown which present a "branching region". In the nanocrystal of Figure $3 d$, the central region between the two lobes can be indexed as the (214) zone axis (or (1014) in the Miller-Bravais notation) of the wurtzite structure. Also, the two arms have wurtzite structure, and therefore the whole nanocrystal represents a multiple twin.

At a molar ratio of MPA to ODPA as low as 0.026 (corresponding to a weight percentage of MPA in the mixture of phosphonic acids of less than 1\%) the nucleation rate
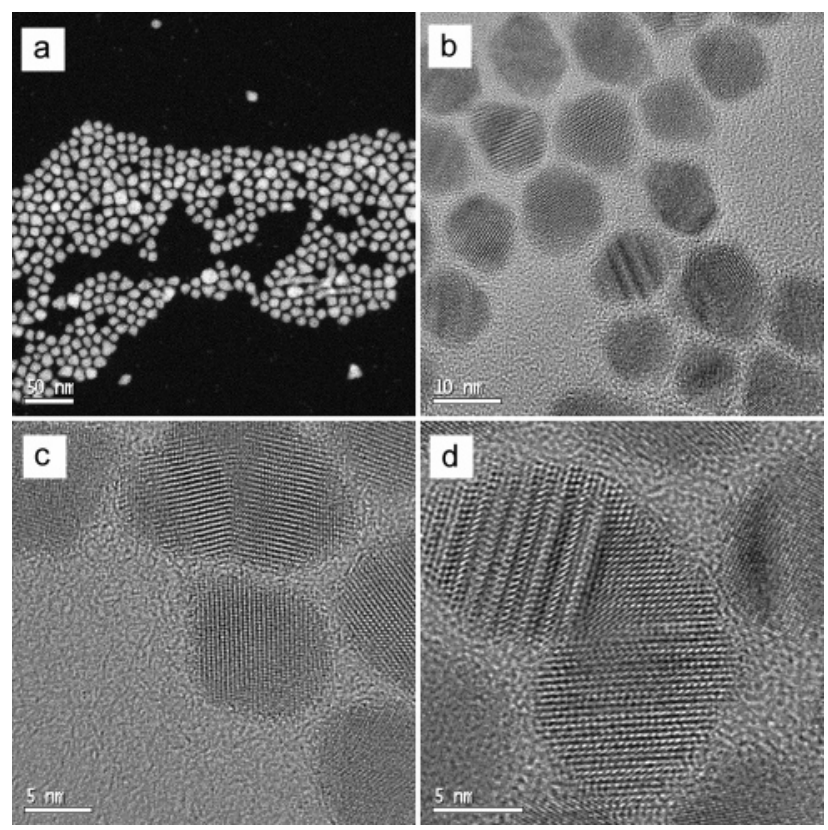

Figure 3. (a) Low-magnification HAADF image of nanocrystals grown in the absence of MPA, which shows that the shapes of most clusters are roughly isotropic and that there is no contrast variation inside them. $(b-d)$ HRTEM images of some representative nanocrystals, highlighting the presence of stacking faults (b), and of occasional wurtzite twins (c, d).
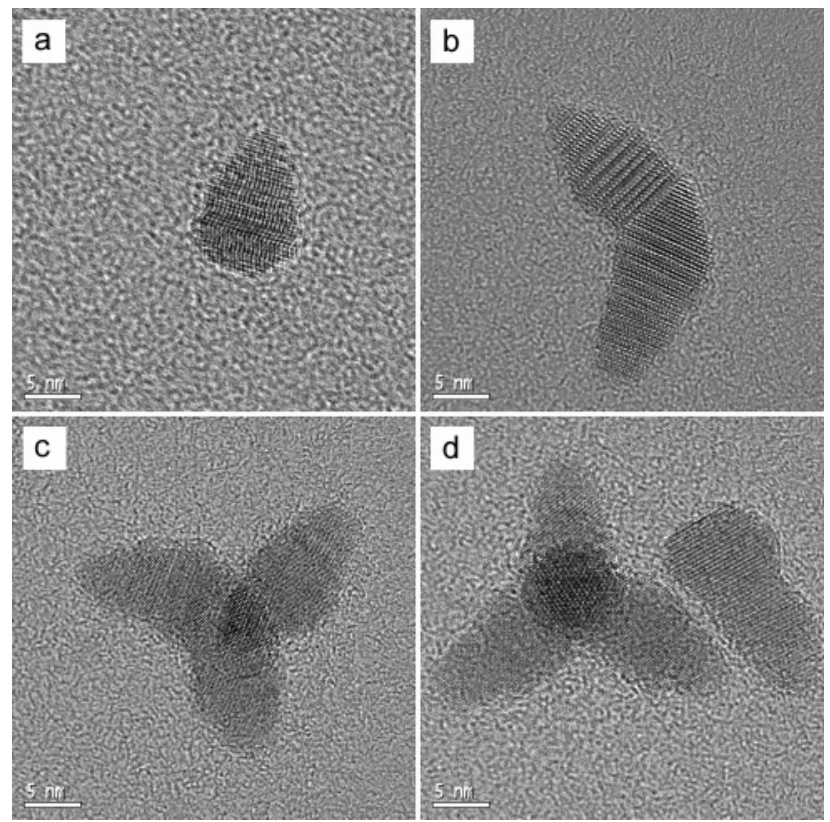

Figure 4. HRTEM images of some representative nanocrystals of the sample grown at MPA:ODPA molar ratio equal to 0.026 (see also Figure $2 b)$. In the branched nanocrystals shown in (c, d) the spacings in the arms can be compatible with the sphalerite structure, except for the arm pointing upward. It is very unlikely that the structure of this fourth arm is different from that of the other arms in both nanocrystals, whereas it is more plausible that the peculiar spacing of the wurtzite structure for the arms touching the substrate are not clearly distinguishable under this orientation.

increased with respect to the previous synthesis, as a color change of the solution from pale yellow (the color of the injected Te:TOP solution) to brown (nanocrystals) occurred much earlier after the injection. The synthesis yielded mainly anisotropic nanocrystals with pearlike shapes, along with dipods, tripods and some tetrapods (Figure $2 \mathrm{~b}$ and Figure 4). These nanocrystals were either single wurtzite domains or were made by wurtzite 


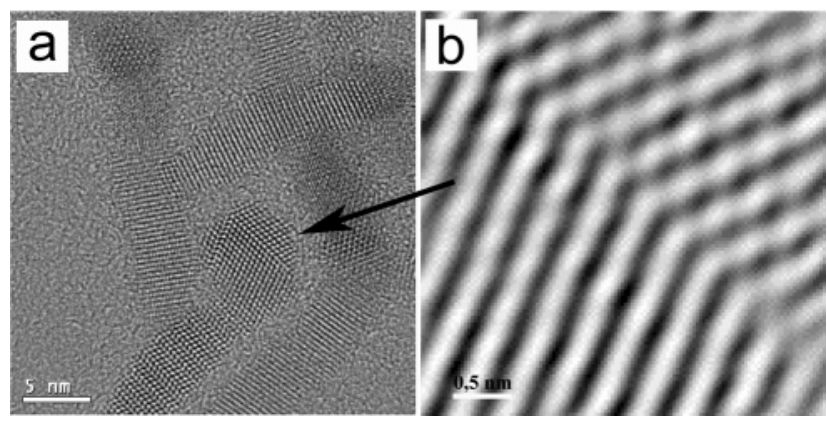

Figure 5. (a) Matchstick-shaped nanocrystal revealing several twins in the head of the matchstick. The region indicated by the arrow is shown in (c) at higher magnification. This area is close to the [1100] zone axis. The defect contrast can be identified as a $(11 \overline{2} 2)$ twin boundary, with a headto-tail configuration of the crystal polarity. The arrangement of the atoms close to the boundary corresponds to the one shown in the model of Figure $1 d$.

twins (Figure 4). In all cases the domains were elongated along the $c$ axis. The presence of sphalerite nanocrystals was very seldom observed. This in addition indicates that the presence of MPA decreased the probability of formation of sphalerite phase considerably.

At higher MPA:OPDA ratios, the branching increased further, as well as the anisotropic growth (Figure $2 \mathrm{c}-\mathrm{g}$ ). Ratios in the range $0.08-0.09$ yielded mainly tripods and tetrapods, whereas nanocrystals with multiple branching points dominated at ratios higher than 0.10 .

X-ray diffraction spectra of the branched samples (for instance, the samples shown in Figure 2d and 2f, see Supporting Information), resembled in their shape those of wurtzite nanorods. ${ }^{17,43}$ They show, in fact, a high degree of distortion of the intensity ratios due to shape anisotropy, in particular with an enhancement of the reflections associated with crystalline domains along the $c$ axis of the wurtzite structure.

Extensive HRTEM observations were carried out on nanocrystals. To uncover the nature of the branching in tetrapods, it would be desirable to image the central region. Unfortunately, such a direct observation is often made difficult by the presence of the arms, which hinder most of this region. A more straightforward analysis can be carried out on structures missing several arms, such as tripods, dipods (Figures $3 \mathrm{~d}$ and $4 \mathrm{~b}$ ), or matchstickshaped nanocrystals, often present together with branched nanocrystals (Figure $2 \mathrm{c}-\mathrm{e}$ ). These latter structures are characterized by the presence of easily identifiable twinned domains, of which only one has been able to grow anisotropically and has developed to an arm. Therefore, the twinned region is always localized on one side of the CdTe rod, and so it forms the head of the resulting matchstick shape. One of these matchsticks is shown in more detail in Figure 5a. In particular, the region indicated by the arrow is close to the [1100] zone axis. The twin of interest can be seen at higher magnification at Scherzer defocus in Figure 5b. Here, the defect contrast is the same as the one reported recently by Yan et al. for wurtzite $\mathrm{ZnO}^{42}$ and can be identified as a (11/22) twin boundary. The different positions of the fringes at the opposite sides of the twin boundary indicate that the two domains are not mirror images of each other, but instead the arrangement of the atoms corresponds to the one shown in the model of Figure 1d. Therefore, this is a

(43) Peng, X. G.; Manna, L.; Yang, W. D.; Wickham, J.; Scher, E.; Kadavanich, A.; Alivisatos, A. P. Nature 2000, 404, (6773), 59-61.
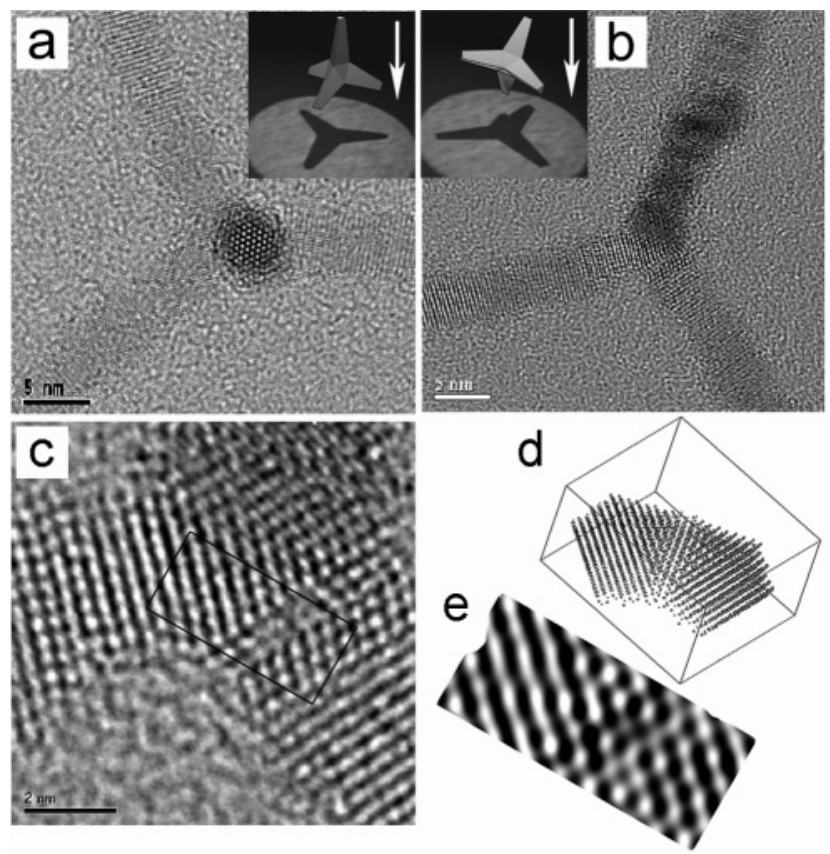

Figure 6. (a) HRTEM image of a CdTe tetrapod with one arm pointing upward. (Inset) Orientation of the nanocrystal with respect to the electron beam (white arrow). The analysis of the symmetry and of the lattice spacing of the arms of several tetrapods indicates a wurtzite structure (space group $\left(\mathrm{P6}_{3} \mathrm{mc}\right)$ with lattice parameters corresponding to $a=0.458 \mathrm{~nm}$ and $c=$ $0.75 \mathrm{~nm}$, respectively). (b) Tilted tetrapod. (Inset) Orientation again of the nanocrystal with respect to the electron beam (white arrow). In particular, in this geometry two arms are superimposed. (c) Central region of the tetrapod shown in (b) is imaged here at higher magnification. The rectangular contour is the region that is the object of the image simulation. (d) Supercell containing the atomistic model used to simulate the HRTEM image of the central region of the tetrapod, viewed along its $\overline{247}$ zone axis and rotated around this axis (which is perpendicular to the plane of the image) so that the model has the same orientation as the real nanocrystal in the HRTEM image in (c). (e) Simulated HRTEM image of the core region of the tetrapod contoured in (c).

$(11 \overline{2} 2)$ twin boundary with a head-to-tail configuration of the polarities of the two wurtzite domains.

Branching in CdTe nanocrystals is occurring only if more than two wurtzite domains are present in the nanocrystal, such as for instance in the dipod shown in Figure 3d. In that case, the central wurtzite domain is a "slow growing" domain (as discussed in the Introduction), and this is attached to "two fast growing" wurtzite domains. Therefore, at least two twin planes are present in a branched nanocrystal (in this case the branched nanocrystal is a dipod). Unfortunately, the visualization of these planes from a favorable zone axis is made difficult by the orientation of the nanocrystal with respect to the electron beam, and a tilting experiment rarely improves this view.

In the case of a tetrapod, a view of the central core region can be obtained by tilting the particle from its natural orientation on the substrate (which is the one with one upstanding arm as in Figure 6a), so that its TEM image is, for instance, that shown in Figure 6b (the core region is seen at higher magnification in Figure 6c). Due to the small size of the structures studied here, it is not possible to align precisely a convenient zone axis, but it is only possible, with great efforts, to tilt the region of interest close to a zone axis with an uncertainty of a few degrees. In the present case, the two arms that point downward are as sketched in the model shown in the inset of Figure 6b. In such a nanocrystal, the geometry of the HRTEM experiment is more 
complicated with respect to the case shown in Figure 5, and to understand the HRTEM image contrast it is necessary to have a comparison with the relevant simulated image. Hence, a simulation of the HRTEM image of the central region between the two lower arms was carried out (Figure 6e). The atomistic model (Figure 6d) was built by joining three hexagonal domains sharing the $(11 \overline{2} 2)$ twin boundary, the same as the one present in the matchsticks of Figure 5. Two domains represent the arms, joined to a central domain. The atomistic model used for this purpose is actually a dipod, as this contains the minimum number of twins necessary to model the region of interest (in this region, the electron beam would cross only these three domains). As can be seen from Figure 6e, the calculated HRTEM image qualitatively reproduces the experimental image. Considering the supercell structure used to model this image (Figure 6d) the HRTEM image has been successfully simulated by considering such supercell as oriented along its [ $\overline{2} \overline{4} \overline{7}]$ zone axis. In this projection, the domain on the left (the left arm) is hiding part of the central domain. It is worth noting that this simulation shows how a tilt of the structure produces a not intuitive image of the tetrapod and in particular of the twinned region. Therefore, the way to proper understand a HRTEM image contrast of such a complex object is to compare the experimental image to the relevant simulation.

The occurrence of twins in CdTe must be promoted if their energy of formation is low, or if the growth environment contributes to lower such energy of formation. Although several types of wurtzite twin planes have been observed in other materials (such as $\mathrm{ZnO}^{5}$ ) and are likely to be present also in CdTe, we choose to focus on the $(11 \overline{2} 2)$ twin boundary. Our LDA-DFT calculations show that in bulk CdTe the energy of formation for the $(11 \overline{2} 2)$ twin boundary with a head-tail polarity configuration is of the order of $70 \mathrm{~mJ} / \mathrm{m}^{2}$, which is intermediate between that of $\mathrm{ZnO}\left(40 \mathrm{~mJ} / \mathrm{m}^{2}\right)$ or $\mathrm{InN}\left(51 \mathrm{~mJ} / \mathrm{m}^{2}\right)$ and that of other wurtzite semiconductors, such as AlN and GaN (109 and $107 \mathrm{~mJ} / \mathrm{m}^{2}$, respectively), as reported by Yan et al. ${ }^{42}$ Therefore, (11) 2 ) twinning should not form in CdTe easily, unless the presence of an additional perturbation could promote somehow its formation. This seems to be the effect of adding MPA in our syntheses, together with the enhancement of anisotropic growth (these two features could be interconnected). The possible roles of MPA in this process are highlighted in the discussion section. We point out that $(11 \overline{2} 2)$ twin boundaries, with tail-to-tail or head-to-head configuration would have a much higher energy of formation, in analogy with other materials, ${ }^{42}$ as they would involve the formation of unsaturated bonds and the build-up of considerable strain at the twin interface. The occurrence of these types of twins in CdTe would be justified if we could find dipods with only one twin plane (i.e. two fast growing wurtzite domains joined together). This twin plane would be localized at the center of the branching region of these dipods. However, we did not find such types of dipods in our samples.

\section{Discussion}

The synthesis of colloidal CdTe nanocrystals in a mixture of surfactants highlights the intrinsic complexity of the crystal growth process and how this can be influenced by the presence of impurities. Several reports on the growth of CdTe tetrapods have appeared recently. ${ }^{18-20}$ In one of them, ${ }^{20}$ tetrapods of varying arm length and diameters were synthesized in a mixture of TOPO, TOP, and ODPA. Later, we found out that the commercially available ODPA contained, until recently, various impurities. ${ }^{44}$ When we performed the synthesis of CdTe nanocrystals in a purer ODPA (99\%, prepared by Polycarbon Industries according to a new protocol), we could obtain only roughly spherical nanocrystals (Figures $2 \mathrm{a}$ and 3 ). The series of experiments reported in this work, in which MPA is present at a known concentration in the mixture of surfactants, reveals that the active component that controls branching and anisotropic growth can be a phosphonic acid with a short alkyl chain. We cannot exclude that other molecules, as for instance alkyl phosphonic acids with intermediate chain lengths, or closely related molecules, might have comparable effects. As an example, syntheses performed with propylphosphonic acid yielded similar results (data not shown).

On the basis of the experimental evidence, we are confident that twinning is responsible for the branching of CdTe nanocrystals. The nanocrystal shapes observed in our experiments indicate in fact that, the higher the concentration of MPA in the growing medium, the higher the average number of twins in the starting nuclei will be, and also the more anisotropically the nanocrystals will grow. Following these observations, we can propose a model that explains the branching behavior. According to this model, which is sketched in Figure 7, the number of arms formed in the nanocrystals depends on the number and on the type of twins present in the starting nucleus. When no MPA is present, twinning has low probability of occurrence. Also, the formed nuclei will grow more or less isotropically, yielding roughly spherical or faceted nanocrystals (as for instance in Figure 2a). At low concentrations of MPA, the nuclei either will be still single domains or will be formed mainly by two wurtzite domains sharing a twin plane (Figure 7a). Both types of nuclei will expose, in addition to other facets, one polar (0001) facet and one polar $(000 \overline{1})$ facet. Of these two polar facets, one will grow much faster than the other, resulting in a pear- or matchstick-shaped nanocrystal (Figure 7a). These shapes can be seen in most nanocrystals of Figure $2 b$ (pears) and Figure 2c (matchsticks). At higher concentrations of MPA, the nuclei will be formed by a higher number of twins (Figure $7 b, c)$. Therefore, these nuclei will show more than one fast growing facet and so will develop more arms, becoming for instance a dipod (Figure 7b), a tripod, or a tetrapod (Figure 7c). At even higher concentration of MPA, twinning will be favored not only at nucleation but also during growth. In these cases, each arm can branch further, as in the hyper-branched structures of Figure 2f,g.

We would like to stress that the proposed mechanism, with perfectly shaped and nicely matching domains, all developing boundaries at the same time (as shown in Figure 7), represents a highly idealized view of the structure of the initial nucleus and of the overall shape evolution. More irregular shapes, often observed in our samples, indicate that the actual branching mechanism is likely to be more complex than the one depicted here. It is quite likely that additional branching pathways exist, which are based on the formation of multiple twins other than those due to the $(11 \overline{2} 2)$ type. This is particularly true for nanocrystals in which there is a high level of branching. In addition, we cannot exclude here the formation of branched

(44) See the Supporting Information for the description of impurities present in ODPA. 
a)

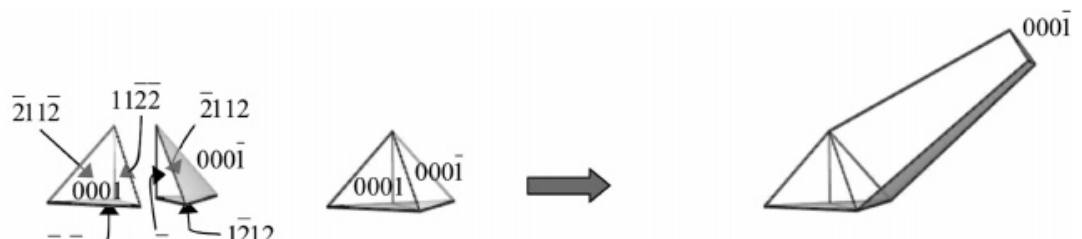

b)
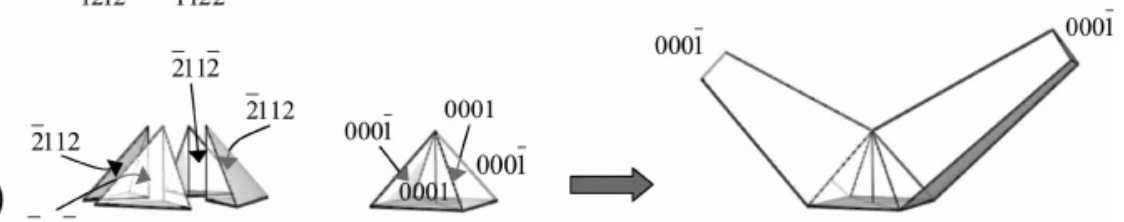

C)
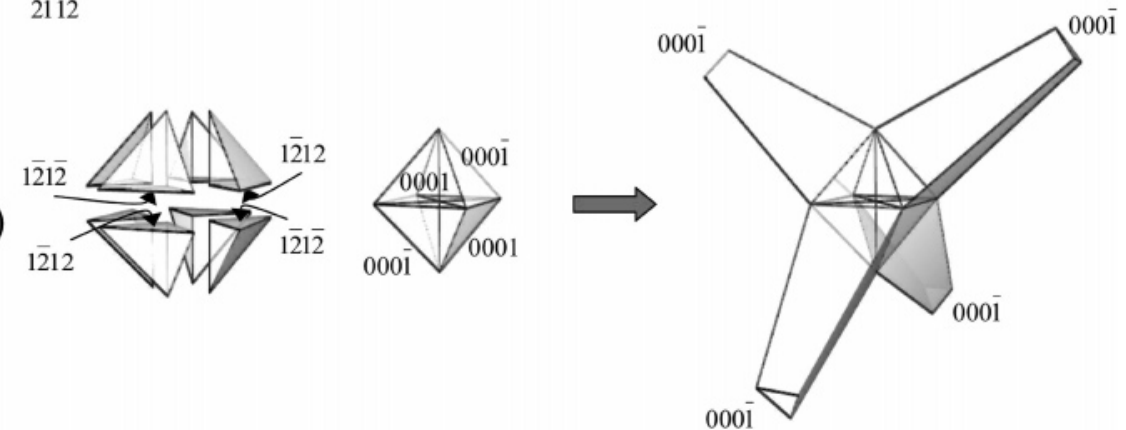

Figure 7. Sketch of the multiple-twin model. The left column shows an exploded view of three different types of nuclei (middle column) that could rationalize the formation of rods (a), dipods (b), and tetrapods (c). Two types of domains are considered in this model (as shown in a, left side). One domain exposes a (0001) facet (we call it type I domain), whereas the other domain exposes a (0001) facet (type II domain). In a multiple-twin nanocrystal the two types of domains are connected to each other by a twin boundary. Of these two types of domains, only one can grow to an elongated structure, although our present knowledge does not allow us to identify which one of the two domains this will be. To help the reader understand the model better, we assume here that the "fast growing" domain is of the type I. Therefore, the nucleus from which a rod (or a matchstick) evolves is composed of at least one domain which is of type I. This domain can be joined to one type II domain (as shown in a) or to more type II domains (up to three) by (1122) twin boundaries. These will be the slow growing domains. Then, if a second twin boundary is generated between any of these type II domains and yet a new type I domain, a second arm will form (a possible case for this situation is shown in b). In the most crowded situation, eight domains can join together to form an octahedral crystal (as shown in c). Four of these domains can be of type I, and four of type II, and in this case four arms will branch out from this core, forming a tetrapod.

structures also based on the occurrence of wurtzite-sphalerite polymorphic modifications.

A small molecule such as MPA can play different roles in the growth kinetics of CdTe nanocrystals. We can try to elucidate these possible roles based on our experimental results and on previous work on nanocrystals. First of all, the $\mathrm{Cd}$ precursor in solution is a complex of $\mathrm{Cd}^{2+}$ with phosphonate molecules. ${ }^{45}$ Therefore, the higher the concentration of MPA relative to that of ODPA, the more likely it will be for $\mathrm{Cd}^{2+}$ to be bound to methyl phosphonates. This sort of complex will be much more mobile than the bulkier complex formed by $\mathrm{Cd}^{2+}$ with octadecylphosphonate molecules, and in addition the $\mathrm{Cd}^{2+}$ ions will be less hindered and so more reactive (in other words they will be more available). This accounts for the observed increase in nucleation and growth rate of the nanocrystals at increasing concentration of MPA. It can also account for the elongated growth that is observed in the nanocrystals. Looking at the series of TEM images from Figure 2, a-c, it is clear that long arms are formed at higher concentrations of MPA. Anisotropic growth, in fact, can be sustained only if there is fast supply of monomers from the bulk of the solution. ${ }^{46-48}$ In addition, crystal defects are generated more easily at high growth rates, ${ }^{49}$ and therefore the presence of MPA could also rationalize the much higher probability of formation of twins, which are a

(45) In the synthesis, CdO dissolves in the mixture of surfactants via decomposition by alkylphosphonic acids. The reaction leads to the formation of a cadmium phosphonate complex and releases water as a byproduct.

(46) Peng, Z. A.; Peng, X. G. J. Am. Chem. Soc. 2001, 123, (7), 1389-1395.

(47) Peng, Z. A.; Peng, X. G. J. Am. Chem. Soc. 2002, 124, (13), 3343-3353.

(48) Peng, X. G. Adv. Mater. 2003, 15, (5), 459-463. particular type of planar defect. This behavior is consistent with earlier observations on a similar system $\left(\mathrm{CdSe}\right.$ nanocrystals $\left.{ }^{47}\right)$, in which an increase in the concentration of precursors resulted in higher nucleation and growth rates of nanocrystals, but also promoted the formation of multibranched nanocrystals.

Another (or an additional) mechanism by which we can imagine that the presence of MPA induces twinning could be a templating effect mediated by the MPA molecules that are passivating the surface of the nanocrystals during nucleation and growth. In other words, it might possible for these MPA molecules to promote an arrangement between the atoms on the surface of the crystal and those approaching the surface from the solution, which could favor the formation of a twin boundary. To decide by which mechanism the formation of twins occurs, extensive studies would be necessary, which go beyond the scope of this work.

\section{Conclusions}

In this work, we have reported a simple and robust protocol for the synthesis of CdTe nanocrystals with a good control over their branching. Detailed HRTEM experiments and HRTEM image simulations have revealed that the branching region is characterized by the presence of multiple twins, among which the $11 \overline{2} 2$ twin boundary with a head-to-tail polarity configuration was identified in matchstick-shaped nanocrystals. The presence of this type of twin supports the tetrapod shape on the light of

(49) Markov, I. V. Crystal Growth for Beginners: Fundamentals of Nucleation, Crystal Growth, and Epitaxy; World Scientific: Singapore, 2003. 
the multiple-twin model discussed above and facilitates the rationalization of other shapes obtained in these types of syntheses, such as matchsticks, dipods, tripods, and multibranched nanocrystals. The formation of these shapes would be difficult to explain by the model based on a cubic sphalerite nucleus. A sphalerite nucleus exposes four equivalent, fast growing facets, and therefore this type of object would naturally evolve into a tetrapod shape. There is apparently no reason why only some of these four equivalent, fast growing facets, would grow much faster than the others, generating structures with a number of arms lower than four.

We found that twinning, as well as anisotropic growth, can be favored by the presence of suitable molecules, such as MPA. We have also drawn some hypotheses on the possible microscopic mechanisms that trigger the formation of twins and, consequently, of branched nanocrystals. Additional studies (both theoretical and experimental) by our group on this matter are in progress and will be the subject of a future work.

Acknowledgment. This work was supported in part by the European project SA-NANO (Contract Number STRP 013698), by the Italian MIUR 297 project (Contract Number 13587) and by the German research foundation (DFG, Emmy Noether program). For the calculations, this work used the resources of the U.S. National Energy Research Scientific Computing Center at NERSC (U.S. Department of Energy under Contract No. DEAC03-76SF00098). We acknowledge Dr. Yanfa Yan (National Renewable Energy Laboratory, Golden, CO 80401, U.S.A.) for having given us the geometrical details of the $\mathrm{ZnO}$ supercell which we adapted for our calculations on CdTe. We would like to acknowledge Professor A.P. Alivisatos (UC Berkeley) and Dr. L.W. Wang (NERSC Berkeley) for many inspiring discussions.

Supporting Information Available: Description of the impurities often present in ODPA; XRD characterization of spherical and branched nanocrystals; geometrical details of the computations (derivation of cell parameters and atomic positions for the twin formation energy; atomistic model of the multipletwinned region used for the simulation of the HRTEM image). This material is available free of charge via the Internet at http://pubs.acs.org.

JA054893C 